\title{
Lighting spectra for the maximum colorfulness
}

\author{
Osamu Masuda $^{* a}$, João M. M. Linhares ${ }^{a}$, Paulo E. R. Felgueiras ${ }^{a}$, Sérgio M. C. Nascimento ${ }^{\text {a }}$ \\ ${ }^{a}$ Center of Physics, Gualtar Campus, University of Minho, Braga, 4710-057 Portugal
}

\begin{abstract}
The advent of modern solid-state sources enabled almost any spectrum for lighting and a wide range of possibilities in color rendering. The quality of the lighting has been typically evaluated by the color rendering index which measures how much the colors of objects illuminated by the light under test look similar to those produced when the objects are illuminated by the daylight or a conventional incandescent light. On the other hand, how colorful or vivid the colors under the illumination are perceived is also an important quality to evaluate lighting. We investigated, computationally, the spectral profiles of the illumination that maximizes the theoretical limit of the perceivable object colors. A large number of metamers with various degree of smoothness were generated using the Schmitt's elements method at chromaticity points on and around the Planckian locus ranging from 2,222 $\mathrm{K}$ to $20,000 \mathrm{~K}$. The general color rendering index (CRI) and MacAdam volumes in CIELAB color space were calculated for each metamer. The metamers maximizing the CRI had smoother spectra than the metamers maximizing the MacAdam volume. These results show that maximum colorfulness in nature can only be obtained with spectrally non-smooth illumination.
\end{abstract}

Keywords: color vision, color rendering, illumination, optimal colors, colorimetry

\section{INTRODUCTION}

The colors of objects are influenced by the spectrum of the illumination. For example, low pressure sodium-vapor lamps are the most efficient electrical light source but everything looks yellow under these lamps and one cannot distinguish any difference of colors other than lightness. The quality of the lighting has been typically evaluated by the color rendering index ${ }^{1}$, which measures how the colors of objects illuminated by the light look similar to those when the objects are illuminated by daylight or a conventional incandescent light. On the other hand, how colorful or vivid the colors under the illumination look like is also an important quality to evaluate lighting and many other quality indices have been proposed ${ }^{2-4}$. The number of discernible colors can be one of the quality indices to evaluate the colorfulness or vividness under the illumination. The theoretically possible object colors with maximum colorfulness under an illumination are called optimal colors. MacAdam calculated the loci of the optimal colors for standard illuminants in the CIE1931 chromaticity diagram ${ }^{5,6}$, and the loci of optimal colors drawn in a three dimensional color space are called MacAdam limits. The number of the discernible colors under an illumination is thought to be proportional to the volume of the MacAdam limit, if the volume was calculated in an ideally uniform color space ${ }^{7}$. Recently, Martínez-Verdú et al. calculated the MacAdam volumes of several illuminants and real lamps in four uniform color spaces ${ }^{8}$. We investigated the spectrum of the illuminant that derives the largest MacAdam color volume by systematically calculating a variety of metamers at wide range of chromaticities on and around the Planckian locus.

\section{METHOD}

\subsection{Chromatic sampling}

Seventeen chromaticity coordinates on the Planckian locus that correspond to the color temperatures ranging from 2,222 $\mathrm{K}$ to $20,000 \mathrm{~K}$ equally spaced in the reciprocal color temperature at an interval of $25 \mathrm{MK}^{-1}$ were chosen as central points. Ten chromaticity points were sampled for each central point on the line perpendicular to the Planckian locus in CIE1960

\footnotetext{
*omasuda@fis.uminho.pt; phone +351-253-604320 (Ext. 605068)
} 
Uniform Chromaticity Scale ranging from -0.01 to +0.01 in chromaticity difference $\mathrm{DC}^{1}$ at an interval of 0.002 . Thus a grid that consists of 187 (17 times 11) points were sampled on and around the Planckian locus as shown in Figure 1.
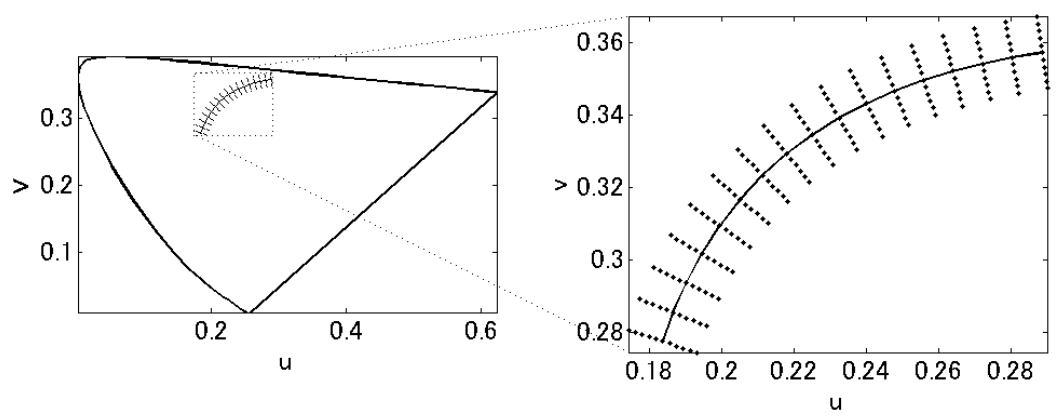

Figure 1. Chromaticities sampled are shown in CIE1960 UCS. The solid line shows the Planckian locus.

\subsection{Metamers}

At each chromaticity of the grid, a number of metamers were generated using the Schmitt's simple element method ${ }^{9}$ for the spectral range of $400-720 \mathrm{~nm}$ with $5 \mathrm{~nm}$ spectral resolution. First, a set of simple elements that have non-zero positive values at only 3 spectral bands was generated. Then second level elements were generated from the simple elements. A second level element was a positive barycentric combination of two simple elements with a randomly determined weighting, and had non-zero positive values at no more than 6 spectral bands. The third level elements were generated in the same way from the second level elements. At each step, 1,000 new metamers are generated, and these steps were repeated until enough metamers with non-zero values at all the spectral bands were obtained. From this large pool of the metamers, we selected 1,000 for each chromaticity so that a uniform distribution with regard to an index $\delta$ (described below) was obtained.

The structure of an illuminant $f_{i}$ was evaluated by a measure of the absolute spectral difference between the illuminant and the equi-energy illuminant E. This measure was defined by Eq. (1)

$$
\delta_{i}=\sum_{k=1}^{M}\left|f_{i, k}-E_{k}\right|
$$

Where $M$ is the number of spectral bands and in this study equal to $65 . f_{i, k}$ is the intensity of the illuminant $f_{i}$ at the $k$ th spectral band. The energy of an illuminant was normalized so that $\sum_{k=1}^{M} f_{i, k}=1 . \quad E_{k}$ is the intensity of the equienergy illuminant $\mathrm{E}$ at the $k$ th spectral band, and is identical to $1 / M$. The more the illuminant $f_{i}$ is spectrally structured, the more the index $\delta_{i}$ approaches 2 . The more the spectrum of the illuminant is flat, the closest the index gets to 0 . The average of the minima of the $\delta$ s of the metamers at each chromaticities was 0.49 , and the average of the maxima was 1.95 . Figure 2 shows examples of metamers at a chromaticity point. 
(a)

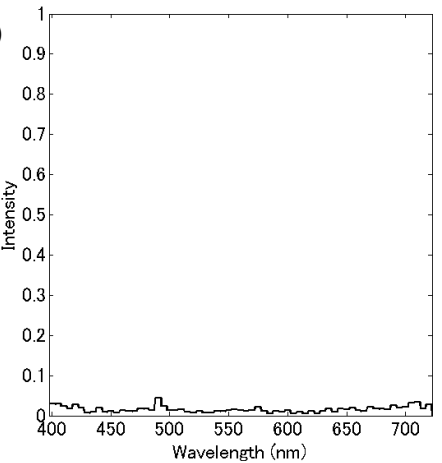

(b)

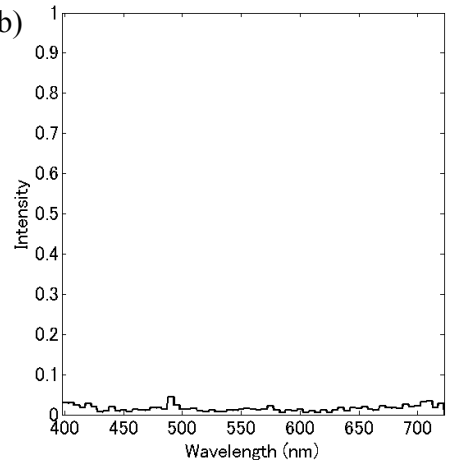

(c)

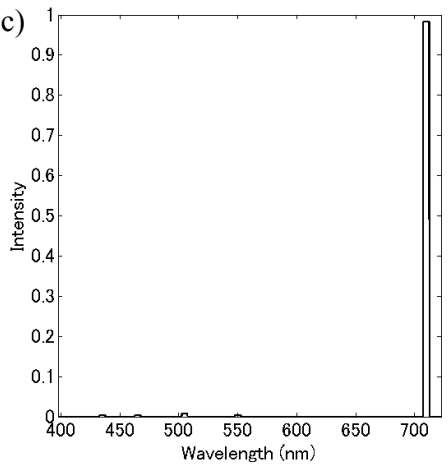

Figure 2. Examples of the metamers at the chromaticity of color temperature $6667 \mathrm{~K}$ on the Planckian locus. (a) Metamer with the smallest $\delta(0.38)$. (b) Metamer with a middle $\delta(1.16)$. (c) Metamer with the largest $\delta(1.93)$. The total energy of a spectrum is normalized to unity.

\subsection{The MacAdam volume}

The volume of the MacAdam color solid was calculated in the CIELAB uniform color space. Nineteen L* levels from 5 to 95 at an interval of 5 were selected for each metamer, and the locus of optimal colors was calculated for each L* level by the method proposed by Masaoka ${ }^{10}$. To calculate the MacAdam color volume, the spectra of the metamers were interpolated from $5 \mathrm{~nm}$ step to $0.1 \mathrm{~nm}$ step rectangularly, and the bands which have zero intensity were substituted by a very small intensity ( $10^{-10}$ times of the total energy). The shift of the chromaticity by this modification of the spectrum was minimal $\left(\Delta \mathrm{E}_{\mathrm{uv}}<0.001\right)$. The volume of the solid was estimated by computing the smallest convex polyhedron containing all of the points of the MacAdam limit loci in the CIELAB color space.

\section{RESULTS}

Figure 3 shows the map of the largest MacAdam color volume at each chromaticity point under investigation. There is little variation along the direction perpendicular to the Planckian locus, but show an explicit convex shape along the Planckian locus. The maximum point was found on the Planckian locus at the color temperature of $5714 \mathrm{~K}$.

Figure 4(a) shows the spectrum of the metamer with the maximum MacAdam color volume among all the chromaticity points under investigation. (b) shows the loci of the MacAdam limits at each of the $\mathrm{L}^{*}$ levels drawn on the CIE1931 chromaticity diagram. (c) shows the MacAdam color volume in the CIELAB uniform color space. The convex hull volume of (c) was $3.02 \times 10^{6}$, and the CRI of this metamer was -24.6.

Figure 5 shows the relationships between the MacAdam color volume and the CRI at this chromaticity point. Data for the other chromaticity points show similar pattern. (a) shows the volume of the MacAdam limit set expressed as a function of $\delta$. The range of volumes increases as $\delta$ becomes larger, that is, the illuminant becomes more structured. The maximum and minimum volumes are obtained for large $\delta$ s. The range of CRI also increases in (b), and the minimum CRI at a $\delta$ becomes smaller for large $\delta$ s, but the maximum CRI does not decline significantly up to the $\delta$ of around 1.7. (c) represents the MacAdam color volume expressed as a function of CRI. The maximum volume is obtained at a negative CRI, and high CRIs can be obtained only with medium volumes, which suggests that high CRI and large volume cannot be fulfilled by the same illuminant.

Figure 6 shows the $\delta$ of the spectrum of the metamer with largest MacAdam color volume at each chromaticity point. In the same way, Figure 7 shows the $\delta$ of the spectrum of the metamer with highest CRI at each chromaticity point. The $\delta$ s of the metamers with large MacAdam color volume were significantly larger than those with high CRIs, which means the metamers with large MacAdam color volume are more structured than those with high CRI. 
Figure 8 shows the spectrum of the metamer with highest CRI at the chromaticity of the correlated color temperature $5714 \mathrm{~K}$ on the Planckian locus. The $\delta$ and CRI of the mean metamer were 0.69 and 97.2, respectively, and the MacAdam color volume was $2.35 \times 10^{6}$.

Figure 9 shows the MacAdam color volume of the metamer with maximum CRI at each chromaticity point. Comparing Figure 9 and Figure 3, the MacAdam color volume can be expanded up to around 30\% by sacrificing the CRI.

In Figure 3, there is no significant difference of MacAdam limit along the direction perpendicular to the Planckian locus. However in Figure 9, the MacAdam color volume becomes larger at chromaticity points with larger DC. When both the CRI and the volume were taken into account at the same time, the optimal chromaticity can be off the Planckian locus. Figure 10 shows the maximum product of the two quality indices, and the maximal point is around where correlated color temperature $(\mathrm{CCT})=4000 \mathrm{~K}$, and $\mathrm{DC}=0.06$.
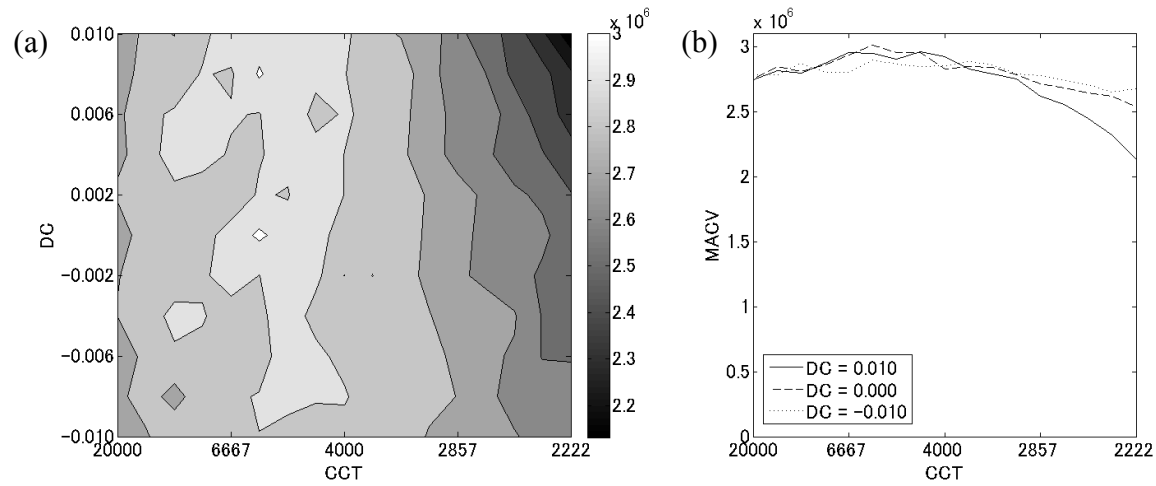

Figure 3 (a) Maximum MacAdam color volume as a two dimensional function of the correlated color temperature (CCT) and the chromatic difference (DC) from the Planckian locus (b) Cross-sectional plot of (a) along lines of DC $=-0.01,0,0.01$.

(a)

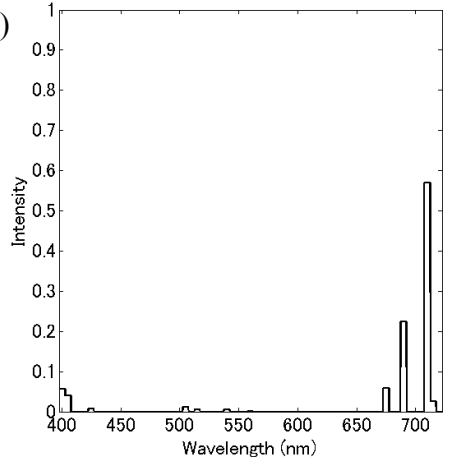

(b)

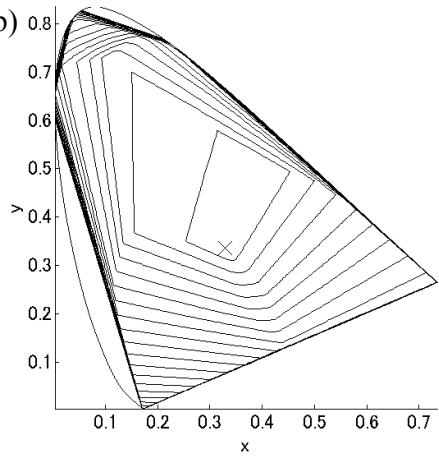

(c)

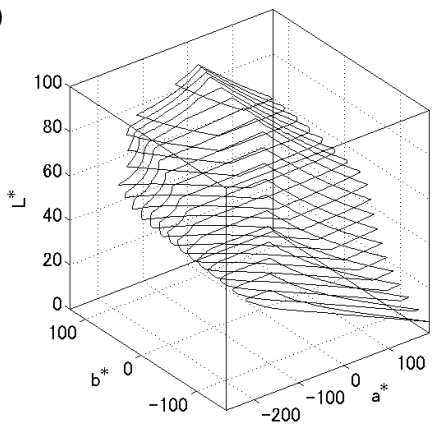

Figure 4. (a) The spectrum of the metamer with the largest MacAdam color volume in the metamer set at the chromaticity of color temperature $5714 \mathrm{~K}$ on the Planckian locus. The $\delta$ and CRI of this metamer were 1.76 and -24.6 , respectively. (b) The loci of the MacAdam limits at each of the $\mathrm{L}^{*}$ levels drawn on the CIE1931 chromaticity diagram. (c) MacAdam color volume with the metamer in (a) in the CIELAB uniform color space. The convex hull volume of this solid was $3.02 \times 10^{6}$. 

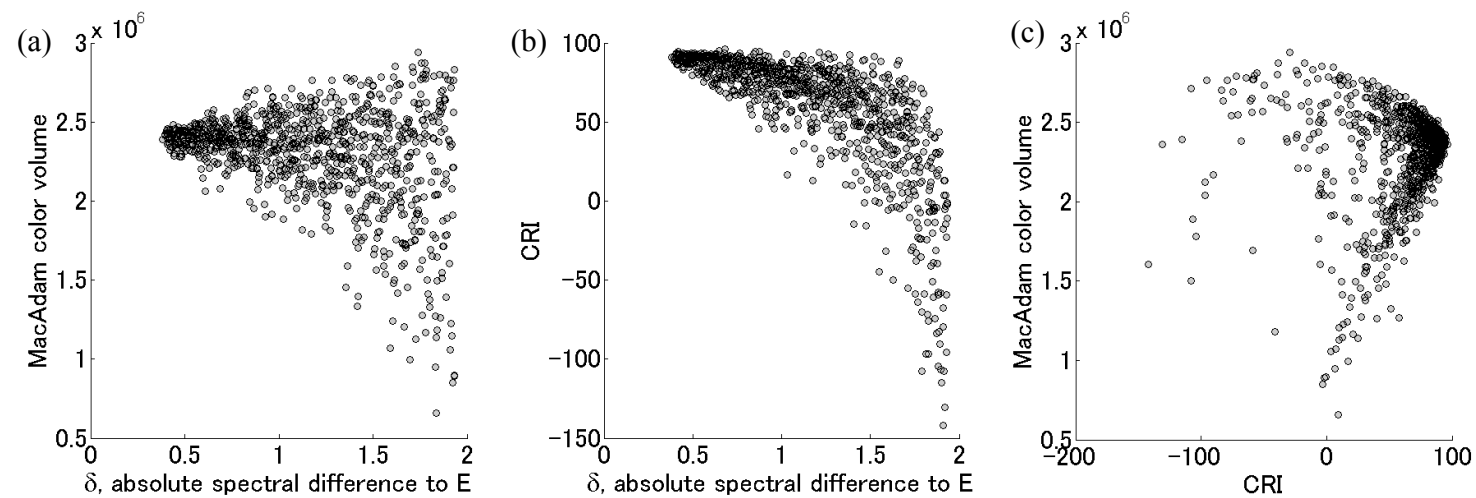

Figure 5. (a) MacAdam color volume obtained with each illuminant expressed as a function of $\delta$. (b) CRI as a function of $\delta$. (c) MacAdam color volume as a function of CRI. Data for a selection of metamers of color temperature $5714 \mathrm{~K}$ on the Planckian locus.

(a)

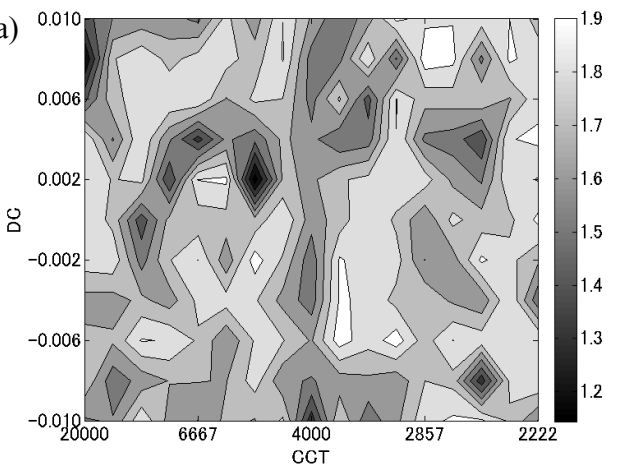

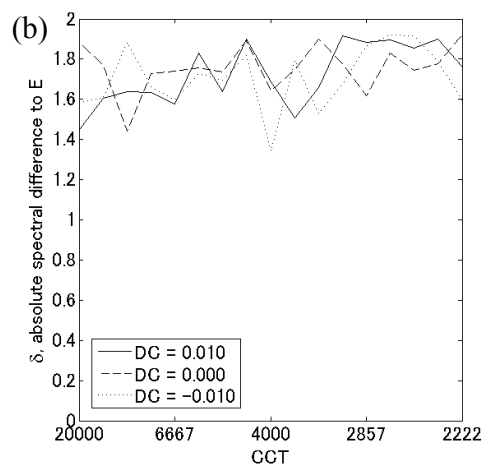

Figure 6. (a) The parameter $\delta$ of the metamer with maximum MacAdam color volume at each chromaticity point. (b) Cross section plot of (a) along lines of $\mathrm{DC}=-0.01,0,0.01$. The mean and the standard deviation of the $\delta$ s were 1.74 and 0.15 respectively.

(a)

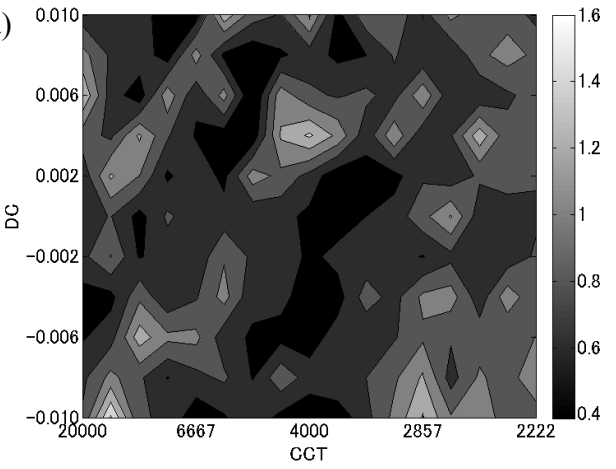

(b)

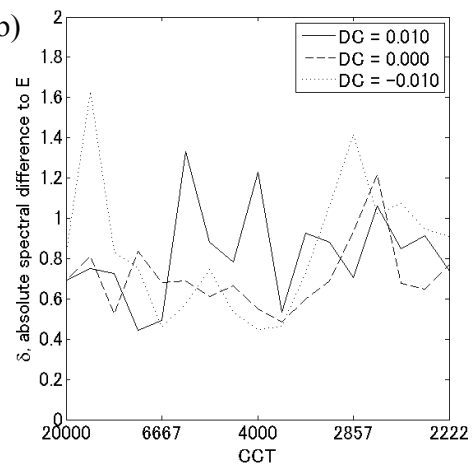

Figure 7. (a) The parameter $\delta$ of the metamer with maximum CRI at each chromaticity point. (b) Cross section plot of (a) along lines of $\mathrm{DC}=-0.01,0,0.01$. The mean and the standard deviation of the $\delta \mathrm{s}$ were 0.80 and 0.23 respectively. 
(a)

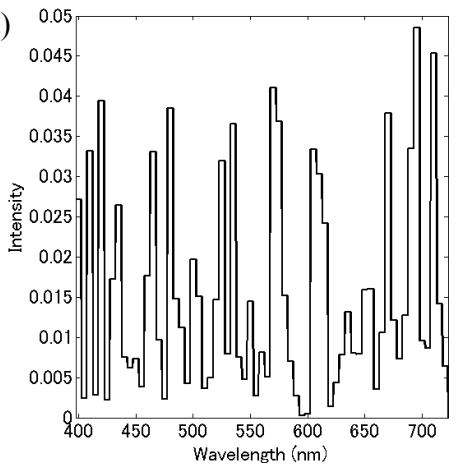

(b)

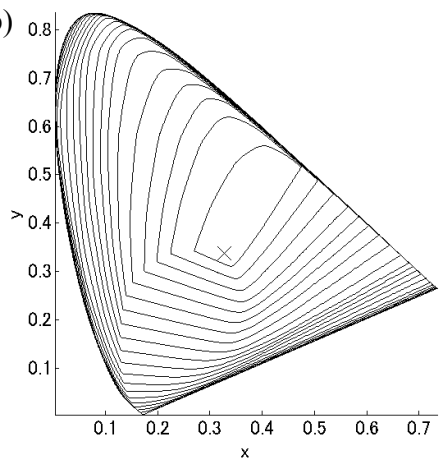

(c)

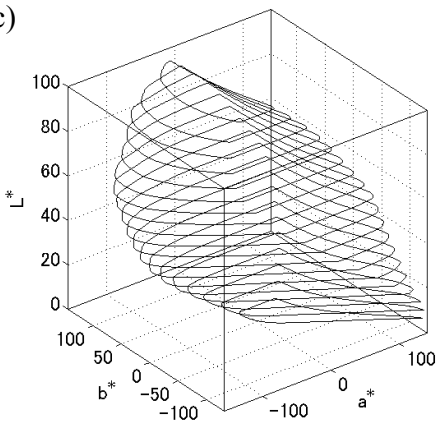

Figure 8. (a) The spectrum of the metamer with maximum CRI in the metamer set at the chromaticity of color temperature $5714 \mathrm{~K}$ on the Planckian locus. The $\delta$ and CRI of this metamer were 0.69 and 97.2, respectively. (b) Loci of the MacAdam limits with the metamer in (a) on the CIE1931 chromaticity diagram. (c) MacAdam color volume with the metamer in (a) in the CIELAB uniform color space. The convex hull volume of this solid was $2.35 \times 10^{6}$.

(a)

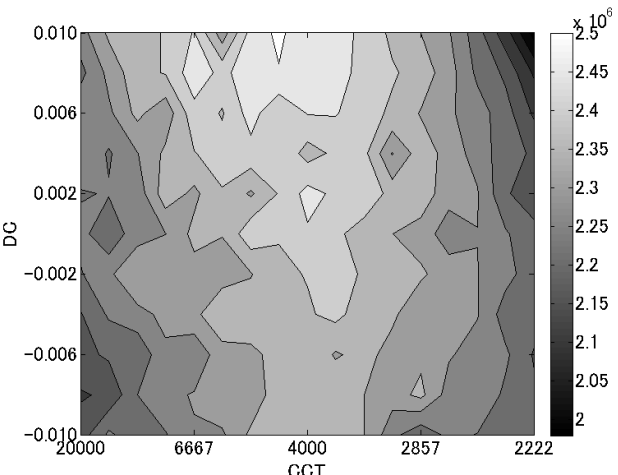

(b)

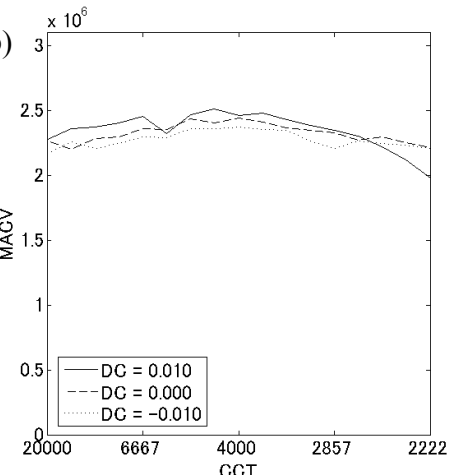

Figure 9. (a) MacAdam color volume of the metamer with maximum CRI at each chromaticity point. (b) Cross section plot of (a) along lines of $\mathrm{DC}=-0.01,0,0.01$.

(a)

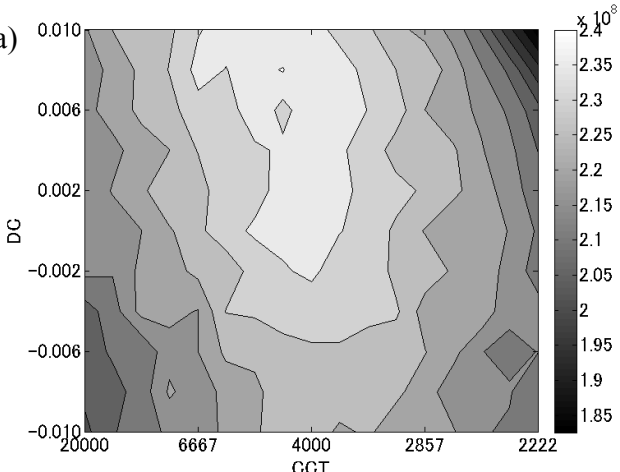

(b)

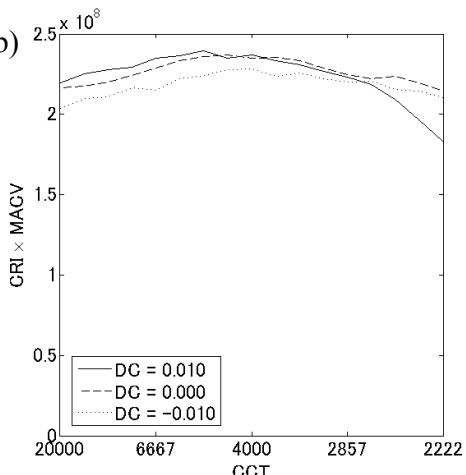

Figure 10. (a) The maximum product of the CRI and the MacAdam color volume at each chromaticity point. (b) Cross section plot of (a) along lines of $\mathrm{DC}=-0.01,0,0.01$. 


\section{DISCUSSION}

In this study we used the MacAdam volumes and sets of metamers with various degree of smoothness to systematically investigate the effects of spectral structure of the illuminant on chromatic diversity. A wide range of chromaticity points on and around the Planckian locus were selected and the general characteristics of the MacAdam volume were revealed systematically under the different sets of metamers. Martínez-Verdú et al. found that the color gamuts of the illuminants with CCT of 5,500 $-10,000 \mathrm{~K}$ are greater than those with CCT outside of this range ${ }^{8}$. This was confirmed in our study for illuminants with a variety of spectral smoothness and wide range of chromaticity.

Comparing the spectra of Figure 4 and Figure 8, the spectrum of the metamer with the largest volume has several peaks in 3 bands at the both ends of the spectrum and at the middle. On the other hand, that of the metamer with the highest CRI has much more peaks all the way along the spectral range, which suggest that high CRI metamers are more structured than large volume metamers. However, between these peaks, the troughs of the spectrum of large volume metamers are zero, but there is no zero intensity band in the troughs of high CRI metamers. The contrast between the peaks and troughs are smaller in high CRI metamers, thus more close to the equi-energy spectrum $\mathrm{E}$.

Comparing Figure 6 and Figure 7, it is obvious that the spectra for large volume and for high CRI are significantly different in their structures, which means that there is no metamer to achieve large volume without sacrificing CRI. Figure 5(c) also endorses this conclusion. However, CRI has been criticized for its validity as a quality index of illumination. We could investigate how much CRI could be sacrificed for larger volume to get preferable illumination for human subject by psychophysical experiments.

In Figure 9, we found that when the CRI is maximized, the MacAdam volume of the spectrum becomes larger at chromaticity points off the Planckian locus in the direction of greenish yellow. The daylight locus is also off the Planckian locus in the same direction, but the DC is around 0.003. As shown in Figure 10, the product of the CRI and the volume also shows the same tendency. If this product is taken as a quality index of the illumination, this index considers that a certain percentage of decrease in CRI is compensated by the same percentage of increase in volume. If the weighting of the volume is more important than CRI for human subjects, the chromaticity of the optimal illumination to reconcile the naturalness and the colorfulness can be off the Planckian locus even more than the daylight locus. This should also be confirmed by psychophysical experiments.

\section{ACKNOWLEDGEMENTS}

This work was supported by the Centro de Física of Minho University, Braga, Portugal and by the Fundação para a Ciência e a Tecnologia (grant PTDC/EEA-EEL/098572/2008). João M.M. Linhares was fully supported by grant SFRH/BD/35874/2007 and Paulo E. R. Felgueiras by grant SFRH / BD / 44698 / 2008.

\section{REFERENCES}

[1] CIE, [Method of Measuring and Specifying Colour Rendering Properties of Light Sources, Publication 13.3], Commission Internationale de l’Eclairage, Vienna (1995).

[2] Judd, B.D.B., "A Flattery index for Artificial Illurninants," Illum. Eng., 62, 593-598 (1967).

[3] Thornton, W. A., "A validation of the colour-preference index," J. Illum. Eng. Soc., 17, 48-52 (1974).

[4] Schanda, J., "A combined colour preference - colour rendering index," Light. Res. Technol., 17, 31-34 (1985).

[5] Macadam, D.L., "The Theory of the Maximum Visual Efficiency of Colored Materials," J. Opt. Soc. Am., 25, 249-

252 (1935).

[6] Macadam, D.L., "Maximum visual efficiency of colored materials," J. Opt. Soc. Am., 25, 361-367 (1935).

[7] Linhares, J.M.M., Pinto, P.D. and Nascimento, S.M.C., "The number of discernible colors in natural scenes," J. Opt. Soc. Am. A, 25, 2918-2924 (2008).

[8] Martínez-Verdú, F., Perales, E., Chorro, E., de Fez, D., Viqueira, V. and Gilabert, E., "Computation and visualization of the MacAdam limits for any lightness, hue angle, and light source," J. Opt. Soc. Am. A, 24, 1501-1515 (2007).

[9] Schmitt, F.J.M., "Method for Treatment of Metamerism in Colorimetry," J. Opt. Soc. Am., 66, 601-608 (1976).

[10] Masaoka, K., "Fast and accurate model for optimal color computation," Opt. Lett., 35, 2031-2033 (2010) 